\title{
Effect of Growth Hormone on Dawn Phenomenon in Healthy Adults
}

\author{
Kuang-Chung Shih ${ }^{1,2}$, Sheng-Hwu Hsieh ${ }^{3}$, Chii-Min Hwu1,4, Ching-Fai Kwok1,4*, \\ Szu-Han Chiu' ${ }^{5}$, Yu-Ching Chou ${ }^{6}$, Low-Tone Ho ${ }^{4,7}$ \\ ${ }^{1}$ Division of Endocrinology and Metabolism, Department of Medicine, Taipei-Veterans General Hospital, Taipei, \\ Taiwan \\ ${ }^{2}$ Division of Endocrinology and Metabolism, Tri-Service General Hospital, National Defense Medical Center, \\ Taipei, Taiwan \\ ${ }^{3}$ Division of Endocrinology and Metabolism, Chang Gung Memorial Hospital, Chang Gung University College of \\ Medicine, Taoyuan, Taiwan \\ ${ }^{4}$ Faculty of Medicine, School of Medicine, National Yang-Ming University, Taipei, Taiwan \\ ${ }^{5}$ Department of Internal Medicine, National Military Taoyuan General Hospital, Taoyuan, Taiwan \\ ${ }^{6}$ School of Public Health, National Defense Medical Center, Taipei, Taiwan \\ ${ }^{7}$ Department of Medical Research, Taipei-Veterans General Hospital, Taipei, Taiwan \\ Email: ${ }^{*}$ fkwok@vghtpe.gov.tw, ${ }^{*}$ tho@vghtpe.gov.tw
}

Received 3 June 2015; accepted 12 July 2015; published 16 July 2015

Copyright $(2015$ by authors and Scientific Research Publishing Inc.

This work is licensed under the Creative Commons Attribution International License (CC BY).

http://creativecommons.org/licenses/by/4.0/

c) (i) Open Access

\section{Abstract}

The purpose of this study was to investigate the involvement of growth hormone in the diurnal variation of insulin sensitivity in healthy adults. Afternoon $(16: 00 \mathrm{hr})$ or night $(23: 00 \mathrm{hr}) \mathrm{pre}$ treatment with a subcutaneous injection of normal saline, human growth hormone to mimic the normal nocturnal rise in growth hormone, or octreotide to inhibit endogenous growth hormone secretion to create a state of relative nocturnal growth hormone deficiency, was given 16 hours before undergoing the modified insulin suppression test in healthy subjects. The morning and evening experiments were separated by an interval of at least 3 days. Thus, each subject was tested on six separate occasions arranged in a random order. A higher value of the steady-state plasma glucose (SSPG) is indicative of lower insulin sensitivity. Plasma glucose, serum insulin, insulin-like growth factor-1, nonesterified fatty acids (NEFA), and metabolic clearance rate of insulin (MCRI) were measured. In the normal saline and human growth hormone groups, SSPG levels were lower in the morning than in the evening. Evening SSPG levels, MCRI, and NEFA concentrations were higher in the participants treated with normal saline and growth hormone than in the octreotide group. Differences in SSPG levels between the morning and evening values were higher in the participants pretreated with normal saline and growth hormone than in those treated with octreotide. A diurnal variation in insulin sensitivity existed in healthy subjects. These results pro- 
vided direct evidence that the role of growth hormone in regulating insulin sensitivity might be related to changes in the MCRI and the metabolism of NEFA in healthy subjects.

\title{
Keywords
}

\author{
Insulin Sensitivity, Dawn Phenomenon, Growth Hormone, Octreotide, Healthy Adult
}

\section{Introduction}

A diurnal variation in glucose tolerance and insulin sensitivity to a glucose load has been reported in human subjects [1]. In addition, glucose tolerance has been reported to be lower and insulin response slower when glucose is given orally either in the afternoon or evening than when it is given in the morning [1]. A similar diurnal variation has been reported in response to an intravenous glucose load [2]. The relatively impaired glucose tolerance observed in the afternoon or the evening is associated with a delayed insulin response to the glucose load, which is probably due to a decreased sensitivity of pancreatic $\beta$ cells to glucose [3].

This "dawn phenomenon" was first described in the 1980s as abrupt increases in fasting levels of plasma glucose, insulin requirement, or both in the early morning between 05:00 and 09:00 hr without antecedent to hypoglycemia. One possible factor affecting this diurnal variation in glucose tolerance is the nocturnal surge in growth hormone [4], even though that surge does not appear to be an important regulator of carbohydrate tolerance in the following morning [5]. Growth hormone reportedly induces insulin resistance in healthy subjects [6]. The serum level of growth hormone shows a nocturnal surge during slow-wave sleep during the night [7]. One study shows that a nocturnal surge in growth hormone secretion was primarily responsible for the diurnal variation in glucose tolerance along with other counter-regulatory hormones such as cortisol, glucagon, and epinephrine [8]. The exact cause of this diurnal variation in plasma glucose levels in healthy subjects has not been established, and nocturnal surges in growth hormone secretion remain a possible explanation. A previous study shows that human growth hormone replacements have beneficial effects on insulin sensitivity in human growth hormone- deficient adults [9]. In another study, time of day was found to influence glucose regulation in healthy subjects [10]. Together, these findings suggest that the diurnal variation in insulin sensitivity may be related to a growth hormone-induced increase in insulin secretion in the early morning for the maintenance of normoglycemia [6] [8] [9]. In contrast to the well-established effects of an excess of growth hormone, little is known about the impact (if any) of physiological changes in plasma growth hormone concentration throughout the day. Growth hormone concentrations are known to vary considerably throughout the day in healthy subjects [4] [11]-[13]; however, the physiological role of growth hormone-induced diurnal variation in glucose tolerance and insulin sensitivity remains unclear.

The purpose of this study was to investigate the role of growth hormone in the dawn phenomenon in healthy subjects that had not previously received other human growth hormone or insulin treatments. The hypothesis was that if growth hormone contributed to dawn phenomenon then the administration of octreotide, a growth hormone secretion inhibitor, would disturb the diurnal variation if it were injected at 23:00 hr. On the other hand, if growth hormone were administered at 16:00 hr, the diurnal variation would be moved forward.

\section{Subjects and Methods}

\subsection{Subjects}

Ten healthy volunteers from the outpatient department of Taipei Veterans General Hospital were enrolled in this prospective experimental study. Subjects were included if they were between 20 and 40 years old of age. Exclusion criteria were: known lactose intolerance, concomitant glucocorticoid therapy, other medications affecting glucose homeostasis, treatment with investigational drugs, the presence of significant diseases or conditions (including emotional disorders or substance abuse) that would likely alter the subject's ability to complete the study, documented gastrointestinal diseases associated with marked digestion or absorption disorders, a condition that may worsen as a result of increased gas formation in the intestine, pregnant/lactating women or women of childbearing age without a medically approved method of contraception, and subjects participating in another 
clinical trial within 90 days of screening. The study protocol was reviewed and approved by the Institutional Review Board of Taipei Veterans General Hospital, Taipei, Taiwan. After explaining the study procedures and protocol to the volunteers prior to physical examination and initiation of the study, written informed consent was obtained from each participant. All included subjects were asked to maintain their eating habits and to record a baseline diet log prior to initiation of the study. Each subject was on a weight maintenance diet of $300 \mathrm{~g}$ carbohydrates daily for more than 3 days prior to enrolment in the study. Two subjects did not complete the study due to nausea, vomiting, and/or diarrhea after treatment with subcutaneous injections of octreotide. Therefore, a total of 8 healthy volunteers completed the study.

\subsection{Protocol Design}

Three timelines show the injection time, fasting duration, and starting time for modified insulin suppression test. The first and second timelines are the studies of morning and evening. English alphabet A (16:00 in Day 1) and C (23:00 in Day 1) indicate the injection time; alphabet B (8:00 in Day 2) and D (15:00 in Day 2) are the starting time for modified insulin suppression test (Figure 1). Three different treatments were administered: 1 ) a subcutaneous injection of $2 \mathrm{~mL}$ of normal saline; 2) a subcutaneous injection of 8 IU of human growth hormone (Saizen, Serono, Switzerland); and 3) a subcutaneous injection of $300 \mathrm{mg}$ of octreotide (somatostatin analogue SMS 201-995, Sandoz, Japan). With half-life of human growth hormone and octreotide, the supraphysical serum concentration level in the modified insulin suppression test is determined. The vehicle is normal saline. The treatments at 16:00 and 23:00 hr were separated by an interval of at least 3 days for each patient. Therefore, each subject received all three treatments at two different times (at both 16:00 and 23:00 hr) arranged in random order, and the tests were conducted $16 \mathrm{hr}$ later on six separate occasions. Fasting plasma glucose (FPG), fasting serum insulin (FSI), insulin-like growth factor-1 (IGF-1), and nonesterified fatty acids (NEFA) concentrations were determined $16 \mathrm{hr}$ after the treatments and prior to performing the modified insulin suppression test.

\subsection{Modified Insulin Suppression Test}

The modified insulin suppression test was conducted by simultaneous infusion of $30 \mathrm{mU} / \mathrm{min} / \mathrm{m}^{2}$ regular insulin, $500 \mathrm{mg} / \mathrm{hr}$ somatostatin, and $6 \mathrm{mg} / \mathrm{kg} / \mathrm{min}$ glucose with three separate infusion pumps for 120 min. Retrograde insertion of the sampling cannula was carried out in a contralateral hand vein. The subject's forearm was placed in a Plexiglas box and heated to $55^{\circ} \mathrm{C}$ to permit sampling of arterialized venous blood as previously described [3]. Blood specimens were collected for measurement of plasma glucose and serum insulin 10 min prior to the start of each infusion and again 0 min before then 30, 60, 90, 100, 110 and 120 min after the start of each infusion. The mean values of plasma glucose at 90, 100, 110 and 120 min were defined as steady-state plasma glucose (SSPG), and serum insulin was defined as steady-state serum insulin (SSSI) [14]. A higher value of SSPG was indicative of lower insulin sensitivity, and a lower value of SSPG was indicative of higher insulin sensitivity.

\subsection{Biochemical Measurements}

Plasma was separated by centrifugation (3000 rpm for 15 minutes) and stored at $-80^{\circ} \mathrm{C}$ until being assayed for FPG, FSI, IGF-1, and NEFA. The FPG level was measured by a glucose analyzer (Model 23A, Yellow Springs

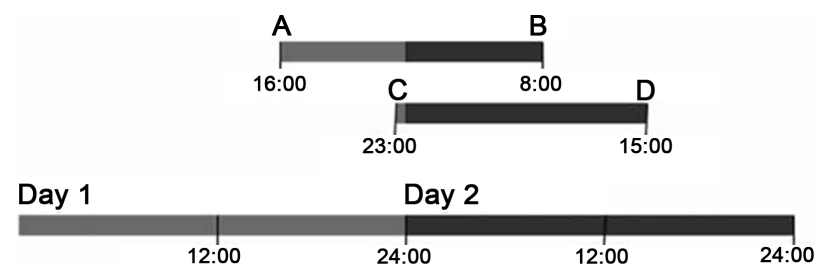

Figure 1. Experimental timeline of the various treatments and modified insulin suppression test. Normal saline, growth hormone, or octreotide were administered subcutaneously at either 16:00 hr (Point A) or 23:00 hr (Point C) on the first day. Sixteen hours later, the effects of those treatments were measured using the modified insulin suppression test at either 08:00 hr (Point B) or 15:00 hr (Point D). On six occasions, separated by an interval of at least 3 days, each subject was treated at 16:00 or 23:00 hr with normal saline, human growth hormone, or octreotide. 
Instrument, OH, USA). FSI was determined by a microparticle enzyme immunoassay using an AxSYM system from Abbott Diagnostics (Abbott Laboratories, Dainabot, Tokyo, Japan). A commercially available immunoenzymetric assay was used to determine IGF-1 levels (Immuno Diagnostic Systems, Boldon, UK). The plasma NEFA levels were determined by fluorometry as described by Noma et al. [15]. The metabolic clearance rate of insulin (MCRI) was calculated as the insulin infusion rate divided by the SSSI level of the modified insulin suppression test, as previously described [10].

\subsection{Statistical Analysis}

Subject age and body mass index (BMI) were expressed as mean \pm standard deviation (SD), and gender was presented as numbers (n). The difference between the morning and evening values was assessed using the paired Student's $t$-test. A $P$ value $<0.05$ was considered statistically significant. Differences between the morning and evening values (evening-morning) were assessed using a one-way analysis of variance (ANOVA) followed by the Bonferroni post hoc procedure. The power calculation for the sample size was supported by Statistics. A $P$ value $<0.017$ was considered statistically significant. Data were expressed as mean \pm SD. All statistical analyses were performed using SPSS version 15.0 statistical software (Chicago, IL).

\section{Results}

Anthropometric data and baseline clinical characteristics have been summarized in Table 1 for all included subjects. Mean BMI was $22.7 \pm 3.5 \mathrm{~kg} / \mathrm{m}^{2}$, and results of the modified insulin suppression test and pharmacokinetic studies in healthy subjects were shown in Table 2. There were no significant differences between the morning

Table 1. Anthropometric data of the eight healthy subjects.

\begin{tabular}{cc}
\hline Gender (M/F) & $4 / 4$ \\
\hline Age (years) & $36.6 \pm 2.5$ \\
BMI $\left(\mathrm{kg} / \mathrm{m}^{2}\right)$ & $22.7 \pm 3.5$ \\
\hline
\end{tabular}

F: female; M: male; BMI: body mass index. Data were summarized as number for gender and mean \pm SD for age and BMI.

Table 2. Results of the diurnal changes of the modified insulin suppression test and pharmacokinetic studies in eight healthy subjects.

\begin{tabular}{|c|c|c|c|c|c|c|c|c|c|}
\hline & \multicolumn{3}{|c|}{ Normal saline } & \multicolumn{3}{|c|}{ Human growth hormone } & \multicolumn{3}{|c|}{ Octreotide } \\
\hline & 08:00 hr & $15: 00 \mathrm{hr}$ & Difference & 08:00 hr & $15: 00 \mathrm{hr}$ & Difference & 08:00 hr & $15: 00 \mathrm{hr}$ & Difference \\
\hline $\begin{array}{c}\text { FPG } \\
(\mathrm{mg} / \mathrm{dL})\end{array}$ & $73.9 \pm 5.5$ & $71.9 \pm 5.7$ & $-2.0 \pm 8.8$ & $79.1 \pm 7.5$ & $80.6 \pm 3.8$ & $1.5 \pm 10.2$ & $79.1 \pm 6.1$ & $79.9 \pm 6.6$ & $0.8 \pm 10.0$ \\
\hline $\begin{array}{c}\text { FSI } \\
(\mu \mathrm{U} / \mathrm{mL})\end{array}$ & $9.0 \pm 4.8$ & $7.4 \pm 5.0$ & $-1.6 \pm 3.3$ & $13.0 \pm 7.5$ & $10.3 \pm 6.1$ & $-2.7 \pm 3.0$ & $11.1 \pm 6.6$ & $10.0 \pm 5.6$ & $-1.1 \pm 2.4$ \\
\hline $\begin{array}{c}\text { MCRI } \\
\left(\mathrm{ml} / \mathrm{min} / \mathrm{m}^{2}\right)\end{array}$ & $643.2 \pm 190.2$ & $691.7 \pm 166.3$ & $48.5 \pm 246.1$ & $669.0 \pm 178.5$ & $671.3 \pm 203.9$ & $2.3 \pm 163.5$ & $645.8 \pm 158.5$ & $534.8 \pm 155.5^{\mathrm{b}, \mathrm{c}}$ & $-111.0 \pm 184.9$ \\
\hline $\begin{array}{l}\text { NEFA } \\
(\mu \mathrm{mol} / \mathrm{L})\end{array}$ & $267.6 \pm 66.7$ & $320.2 \pm 52.6$ & $52.6 \pm 67.3$ & $318.4 \pm 56.6^{\mathrm{a}}$ & $410.0 \pm 67.1$ & $91.6 \pm 72.6$ & $250.6 \pm 56.7^{c}$ & $239.5 \pm 60.1^{\mathrm{b}, \mathrm{c}}$ & $-11.1 \pm 96.3^{\mathrm{c} \dagger}$ \\
\hline $\begin{array}{l}\text { IGF-1 } \\
\text { (ng/mL) }\end{array}$ & $181.5 \pm 43.4$ & $186.3 \pm 46.1$ & $4.8 \pm 37.4$ & $230.9 \pm 38.0$ & $225.0 \pm 44.4$ & $-5.9 \pm 16.3$ & $159.0 \pm 34.1^{\mathrm{c}}$ & $153.9 \pm 36.6^{c}$ & $-5.1 \pm 22.2$ \\
\hline $\begin{array}{l}\text { SSPG } \\
(\mathrm{mg} / \mathrm{dL})\end{array}$ & $116.8 \pm 33.6^{\mathrm{a}}$ & $150.4 \pm 25.3$ & $33.5 \pm 30.6$ & $127.4 \pm 36.3^{\mathrm{a}}$ & $166.2 \pm 23.0$ & $38.8 \pm 28.8$ & $134.6 \pm 30.7$ & $118.4 \pm 35.8^{\mathrm{b}, \mathrm{c}}$ & $-16.2 \pm 26.2^{\mathrm{b} \dagger, \mathrm{c}}$ \\
\hline $\begin{array}{c}\text { SSSI } \\
(\mu \mathrm{U} / \mathrm{mL})\end{array}$ & $50.4 \pm 15.8$ & $46.4 \pm 14.7$ & $-4.0 \pm 22.3$ & $48.7 \pm 16.9$ & $52.0 \pm 16.7$ & $3.3 \pm 11.0$ & $49.4 \pm 14.3$ & $54.8 \pm 13.6$ & $5.4 \pm 9.2$ \\
\hline $\begin{array}{c}\text { BFI } \\
(\mu \mathrm{U} / \mathrm{mL})\end{array}$ & $<2.5$ & $<2.5$ & & $<2.5$ & $<2.5$ & & $<2.5$ & $<2.5$ & \\
\hline
\end{tabular}

FPG: fasting plasma glucose; FSI: fasting serum insulin; MCRI: metabolic clearance rate of free insulin; NEFA: nonesterified fatty acid; IGF-1: insulin-like growth factor-1; SSPG: steady-state plasma glucose; SSSI: steady-state serum insulin; BFI: basal free insulin. Those results were associated with a reduction in SSPG, MCRI and NEFA values in the subjects pretreated with octreotide compared to those receiving normal saline ( ${ }^{\mathrm{b}} P<0.05$ ) and growth hormone ( ${ }^{\mathrm{C}} P<$ 0.05). The differences in SSPG between the morning and evening values (evening-morning) were higher in subjects treated with normal saline ${ }^{\mathrm{b} \dagger} P<0.017$ ) and human growth hormone $\left({ }^{\mathrm{c} \dagger} P<0.017\right.$ ) than octreotide. ${ }^{\mathrm{a}} P<0.05$ (08:00 hr versus 15:00 hr), ${ }^{\mathrm{b}} P<0.05$ (normal saline versus octreotide), and ${ }^{\mathrm{c}} P<0.05$ (human growth hormone versus octreotide) with the paired t-test. ${ }^{\mathrm{b} \dagger} P<0.017$ (normal saline versus octreotide), and ${ }^{\mathrm{c}} P<0.017$ (human growth hormone versus octreotide) with the ANOVA followed and Bonferroni post hoc procedure. 
(8:00 hr) and evening (15:00 hr) SSSI levels following any treatment (Figure 2, upper panel). After administration with either normal saline or human growth hormone, the SSPG levels were lower in the morning than in the evening (Figure 2, lower panel). The evening SSPG levels were higher in the participants treated with normal saline and growth hormone than in those treated with octreotide (Figure 2, lower panel). The differences in SSPG between the morning and evening values (evening-morning) were higher in subjects treated with normal saline and human growth hormone than octreotide (Table 2). There were no significant differences in FPG, FSI, or basal free insulin between subjects treated in the morning and those treated in the evening in any of the three groups (Table 2).

To verify the efficacy of the various treatments, secretion of IGF-1, a downstream effecter of growth hormone was analyzed. Administration of growth hormone at either 16:00 or 23:00 hr resulted in an increase in IGF-1 secretion, suggesting a response to the exogenous growth hormone injection. The injection of octreotide at either 16:00 or 23:00 hr induced a significant reduction in IGF-1 secretion, as shown in Table 2. SSSI was achieved in each treatment (Figure 2, upper panel). Serum IGF-1 levels in the morning and evening were highest in subjects administered human growth hormone, intermediate in those treated with normal saline, and lowest in subjects treated with octreotide (Table 2). Those results suggested a proper execution of the experiments.

To determine insulin sensitivity in the healthy subjects after each treatment, MCRI values were measured by dividing the insulin infusion rate by SSSI concentration. Insulin resistance was determined via SSPI and SSPG. As shown in Figure 2 (lower panel), evening SSPG levels of subjects treated with octreotide were significantly decreased compared to subjects receiving normal saline and growth hormone, suggesting a reduction in insulin resistance. Those results were associated with a reduction in MCRI and NEFA values in the subjects pretreated with octreotide compared to those receiving normal saline and growth hormone. Administration of octreotide at 16:00 hr did not induce a significant decrease in MCRI and NEFA, although there was an increasing trend. Those results suggest that administration of growth hormone caused a reduction in insulin sensitivity (Table 2).

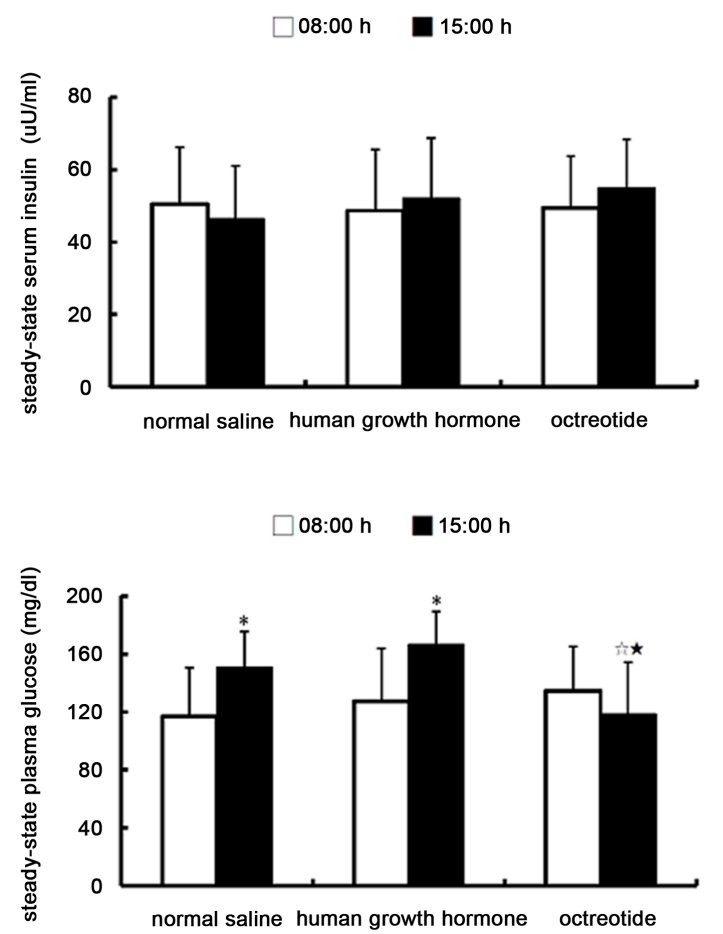

Figure 2. Diurnal changes in steady-state serum insulin (upper panel) and steady-state plasma glucose levels (lower panel) in the eight healthy subjects administered normal saline, human growth hormone, and octreotide. The steady-state serum insulin and plasma glucose levels were obtained at either 08:00 $\mathrm{hr}(\square)$ or 15:00 $\mathrm{hr}(\square)$. After administration with either normal saline or human growth hormone, the SSPG levels were lower in the morning than in the evening $\left({ }^{*} P<0.05\right)$. The evening SSPG levels were higher in the participants treated with normal saline $\left({ }^{\star} P<0.05\right)$ and growth hormone $\left({ }^{\star} P<0.05\right)$ than in those treated with octreotide. Data were expressed as mean \pm SD. $* P<0.05$ (morning versus evening), ${ }^{\star} P<0.05$ (normal saline versus octreotide), and ${ }^{\star} P<0.05$ (growth hormone versus octreotide). 


\section{Discussion}

In this study, subjects were administered exogenous growth hormone to mimic the natural nocturnal surge of growth hormone and the growth hormone secretion blocker, octreotide, to mimic the effect of a loss of function of growth hormone on insulin sensitivity. The effectiveness of those treatments was supported by an increase in IGF-1 secretion when exogenous growth hormone was injected and by the reduction in IGF-1 secretion after administering octreotide. The results showed that a surge of growth hormone induced a reduction in insulin sensitivity in the healthy subjects.

Administration of normal saline resulted in a higher SSPG level in the evening (15:00 hr) than in the morning (08:00 hr). Those results provided evidence of a diurnal variation in insulin sensitivity in the healthy subjects (i.e., that they were more insulin sensitive in the morning). Administration of human growth hormone revealed that the SSPG levels were lower in the morning than in the evening, providing evidence of the diurnal variation in insulin sensitivity (i.e., that they were less insulin sensitive in the evening). That said evening SSPG levels were higher in the participants treated with normal saline and growth hormone than in those treated with octreotide. Differences in SSPG between the morning and evening values (evening-morning) were higher in the healthy subjects treated with normal saline and human growth hormone than in those treated with octreotide, which suggested that octreotide perturbed the biorhythm in the healthy subjects. In addition, the evening MCRI and NEFA levels in the healthy subjects treated with octreotide were significantly lower than in those treated with normal saline and human growth hormone. The effects of growth hormone may therefore be related to changes in MCRI and the metabolism of NEFA in healthy subjects.

A diurnal variation in insulin sensitivity was observed following administration of exogenous normal saline and human growth hormone, which was reversed by injections of exogenous octreotide. In subjects treated with human growth hormone, NEFA concentrations were lower in the morning than the evening and morning NEFA concentrations were higher in those treated with human growth hormone than octreotide. Those results demonstrated that growth hormone perturbed the insulin sensitivity biorhythm and that human growth hormone was associated with increased NEFA levels in healthy subjects. The results of this study suggested that insulin sensitivity was decreased in the morning in healthy subjects compared to the evening, which was likely due to the nocturnal surge of growth hormone in the early morning. This again suggested that the effects of growth hormone might be related to changes in MCRI and the metabolism of NEFA in healthy subjects.

There is a substantial body of evidence linking increases in serum growth hormone concentrations with decreased glucose utilization, indicating that growth hormone may play an important role in the regulation of hepatic glucose production [16]. A sustained increase in serum growth hormone has been shown to cause insulin resistance in healthy subjects [16], subjects with type 2 diabetes mellitus [17], and in rats [18]. A study using radioactive isotopes and insulin clamp techniques demonstrated that improved glucose tolerance after acromegaly treatment could specifically be due to a decrease in peripheral insulin resistance [19]. In another study, it was found that the dawn phenomenon was effectively prevented by applying a bedtime intranasal long-acting somatostatin analog [20].

Adult patients with growth hormone deficiency are insulin resistant, which is probably related to increased adiposity, reduced lean body mass, and impaired physical performance that temporarily worsens when growth hormone treatment is initiated. Conversely, despite an increased lean body mass and decreased fat mass, patients with acromegaly are consistently insulin resistant and become more sensitive after appropriate treatment [21]. It was recently reported that low-dose growth hormone exposure evoked an acute insulin resistance that subsided after $5 \mathrm{hr}$, suggesting that such time-dependent reversibility should be considered when assessing the influence of growth hormone on glucose homeostasis [22]. Nonetheless, although the dawn phenomenon has been attributed to a decrease in insulin sensitivity induced by a nocturnal spike in growth hormone secretion [23], some investigators suggest that an early morning increase in MCRI, rather than a decrease in insulin sensitivity, is the primary cause of the dawn phenomenon [24]. There is also some debate about whether overnight changes in MCRI play a role in the pathogenesis of the dawn phenomenon. Growth hormone is secreted in a pulsatile, episodic manner that is influenced by age, gender, food intake, nutritional status, and physical activity [25]. It has also been suggested that the metabolic effects and MCRI of exogenous growth hormone depend on the time of day of administration [25]. In this study, the influence of these factors on serum triglyceride levels and NEFA concentration were considered, resulting in the decision to measure FPG, FSI, IGF-1, and NEFA concentrations $16 \mathrm{hr}$ after the treatments, prior to performing the modified insulin suppression test. In subjects treated with hu- 
man growth hormone, NEFA concentrations were lower in the morning than in the evening and morning NEFA concentrations were higher in subjects treated with human growth hormone than octreotide. Those findings indicating that human growth hormone was involved in mediation of NEFA levels.

The diurnal variation in insulin sensitivity did not change in the healthy subjects following administration of exogenous human growth hormone. A possible explanation for that finding was that plasma NEFA increased with reduced insulin sensitivity, and that insulin secretion increased with normalized $\beta$-cell function. The results further suggested that fasting hyperglycemia did not occur in the healthy subjects, suggesting that NEFA played a role in determining the degree of insulin sensitivity in healthy subjects. In addition, the results showed that early evening administration of octreotide decreased the MCRI $16 \mathrm{hr}$ later, suggesting a reduction in insulin resistance.

The mechanism (s) behind growth hormone regulation of insulin sensitivity in healthy subjects remains unclear. As with growth hormone, several hormones that play important roles in the regulation of carbohydrate metabolism including catecholamine, glucagon, and cortisol have diurnal rhythms. Theoretically, those diurnal rhythms can result in either an increase in glucose production or a decrease in glucose utilization. Although it has been proposed that an early morning rise in cortisol constitutes a mechanism for increased glucose production, neither metyrapone blockade [26] nor dexamethasone suppression [27] has been shown to abolish the need for an increase in insulin delivery during the dawn period. Thus, glucagon and catecholamines are probably not involved in the pathogenesis of the dawn phenomenon [27].

Biochemically, the effects of growth hormone are complex and much research has focused on the mechanism behind its influence on insulin resistance. A recent study points out that both growth hormone and insulin are key hormones regulating metabolism and growth, each activating different signaling pathways [28]. As such, growth hormone and insulin can interact with each other to regulate cellular metabolism. In turn, it is possible to postulate that growth hormone and insulin can interact directly by signaling “crosstalk” and that insulin regulation of growth hormone signaling is associated with the duration of exposure to insulin. Although evidence suggests that chronic administration of excessive growth hormone can interfere with the activation of specific pathways by insulin [28] [29], several other proteins have also been shown to be involved in the mechanisms underlying growth hormone-induced insulin resistance. For example, Xu and Messina [28] and Domenici et al. [29] show that IGF-1 participates in the control of insulin sensitivity and plays an important role in the hormone balance between growth hormone and insulin.

There are several limitations to this study worthy of mention. First, small sample size in the study is a potential limitation. Second, the study results do not demonstrate any effect of growth hormone on overnight hepatic glucose production, gluconeogenesis, or insulin secretion. In addition, as mentioned above, serum levels of growth hormone are not measured. Growth hormone secretion is pulsatile and growth hormone has a short halflife, yet excess growth hormone is not analyzed. That may in fact be the focus of the impact of physiological changes in growth hormone levels in healthy subjects. Finally, nocturnal lipolysis is not measured in vivo, it only represents an approximation of NEFA. Further studies are needed to confirm the findings of this study.

In summary, the results of this study suggest that insulin sensitivity in healthy subject is decreased in the evening compared with the morning. That reduction in insulin sensitivity may be due to the nocturnal surge of growth hormone in the early morning hours. The effects of growth hormone may be related to changes in MCRI and the metabolism of NEFA in healthy subjects.

\section{Acknowledgements}

The authors would like to thank Drs. Kai-Shun Chen, Ming-Tsung Sun, and Chen-Hao Tsai for reviewing the manuscript and Li-Chuan Hsiao, Yueh-Fen Liu, and Sheng-Hung Li for their excellent administrative assistance. This study was supported in part by funding received from the Tri-Service General Hospital (TSGH-C98-5-S03), Taipei, Taiwan and in part by funding received from the National Health Research Institutes (BS-098-PP-01), Zhunan, Miaoli County, Taiwan.

\section{References}

[1] Malherbe, C., De Gasparo, M., De Hertogh, R. and Hoet, J.J. (1969) Circadian Variations of Blood Sugar and Plasma Insulin Levels in Man. Diabetologia, 5, 397-404. http://dx.doi.org/10.1007/BF00427978

[2] Whichelow, M.J., Sturge, R.A., Keen, H., Jarrett, R.J., Stimmler, L. and Grainger, S. (1974) Diurnal Variation in Re- 
sponse to Intravenous Glucose. British Medical Journal, 1, 488-491. http://dx.doi.org/10.1136/bmj.1.5906.488

[3] Wu, M.S., Ho, L.T., Jap, T.S., Kwok, C.F. and Chen, J.J. (1986) Diurnal Variation of Insulin Clearance and Sensitivity in Normal Man. Proceedings of the National Science Council, Republic of China, Part B, Life Sciences, 10, 64-69.

[4] Bolli, G.B., De Feo, P., De Cosmo, S., Perriello, G., Ventura, M.M., Calcinaro, F., Lolli, C., Campbell, P., Brunetti, P. and Gerich, J.E. (1984) Demonstration of a Dawn Phenomenon in Normal Human Volunteers. Diabetes, 33, 11501153. http://dx.doi.org/10.2337/diab.33.12.1150

[5] Nielsen, M.F., Dinneen, S., Basu, A., Basu, R., Alzaid, A. and Rizza, R.R. (1998) Failure of Nocturnal Changes in Growth Hormone to Alter Carbohydrate Tolerance the Following Morning. Diabetologia, 41, 1064-1072. http://dx.doi.org/10.1007/s001250051031

[6] Fineberg, S. and Merimee, T.J. (1974) Acute Metabolic Effects of Human Growth Hormone. Diabetes, 23, $499-504$. http://dx.doi.org/10.2337/diab.23.6.499

[7] Sassin, J.F., Parker, D.C., Mace, J.W., Gotlin, R.W., Johnson, L.C. and Rossman, L.G. (1969) Human Growth Hormone Release: Relation to Slow-Wave Sleep and Sleep-Walking Cycles. Science, 165, 513-515. http://dx.doi.org/10.1126/science.165.3892.513

[8] Campbell, P.J., Bolli, G.B., Cryer, P.E. and Gerich, J.E. (1985) Pathogenesis of the Dawn Phenomenon in Patients with Insulin-Dependent Diabetes Mellitus. Accelerated Glucose Production and Impaired Glucose Utilization Due to Nocturnal Surges in Growth Hormone Secretion. New England Journal of Medicine, 312, 1473-1479. http://dx.doi.org/10.1056/NEJM198506063122302

[9] Hwu, C.M., Kwok, C.F., Lai, T.Y., Shih, K.C., Lee, T.S., Hsiao, L.C., Lee, S.H., Fang, V.S. and Ho, L.T. (1997) Growth Hormone (GH) Replacement Reduces Total Body Fat and Normalizes Insulin Sensitivity in GH-Deficient Adults: A Report of One-Year Clinical Experience. The Journal of Clinical Endocrinology and Metabolism, 82, 32853292. http://dx.doi.org/10.1210/jc.82.10.3285

[10] Shih, K.C., Ho, L.T., Kou, H.F., Liu, P.C., Hsiao, L.C., Li, S.H., Liu, Y.F. and Wu, M.S. (1992) Diurnal Variation in Insulin Sensitivity in NIDDM Patients and Normal Subjects. Journal of the Formosan Medical Association, 91, 263269.

[11] Yalow, R.S., Goldsmith, S.J. and Berson, S.A. (1969) Influence of Physiologic Fluctuations in Plasma Growth Hormone on Glucose Tolerance. Diabetes, 18, 402-408. http://dx.doi.org/10.2337/diab.18.6.402

[12] Hartman, M.L., Faria, A.C., Vance, M.L., Johnson, M.L., Thorner, M.O. and Veldhuis, J.D. (1991) Temporal Structure of in Vivo Growth Hormone Secretory Events in Humans. American Journal of Physiology, 260, E101-E110.

[13] Butler, P., Kryshak, E. and Rizza, R. (1991) Mechanism of Growth Hormone-Induced Postprandial Carbohydrate Intolerance in Humans. American Journal of Physiology, 260, E513-E520.

[14] Ho, L.T., Chang, Z.Y., Wang, J.T., Li, S.H., Liu, Y.F., Chen, Y.D. and Reaven, G.M. (1990) Insulin Insensitivity in Offspring of Parents with Type 2 Diabetes Mellitus. Diabetic Medicine, 7, 31-34. http://dx.doi.org/10.1111/j.1464-5491.1990.tb01303.x

[15] Noma, A., Okabe, H. and Kita, M. (1973) A New Colorimetric Micro-Determination of Free Fatty Acids in Serum. Clinica Chimica Acta, 43, 317-320. http://dx.doi.org/10.1016/0009-8981(73)90468-3

[16] MacGorman, L.R., Rizza, R.A. and Gerich, J.E. (1981) Physiological Concentrations of Growth Hormone Exert Insulin-Like and Insulin Antagonistic Effects on Both Hepatic and Extrahepatic Tissues in Man. The Journal of Clinical Endocrinology and Metabolism, 53, 556-559. http://dx.doi.org/10.1210/jcem-53-3-556

[17] Shih, K.C., Hsieh, S.H., Kwok, C.F., Hwu, C.M., Hsieh, P.S. and Ho, L.T. (2013) Effect of Growth Hormone on Dawn Phenomenon in Patients with Type 2 Diabetes. Growth Factors, 31, 66-73. http://dx.doi.org/10.3109/08977194.2013.772996

[18] Shih, K.C., Liu, L.Y., Kwok, C.F., Hwu, C.M., Juan, C.C., Hsu, Y.P. and Ho, L.T. (2007) Effect of Reversing Dark-Light Cycles on Normal Diurnal Variation and Related Metabolic Disturbance in Rats. Chinese Journal of Physiology, 50, 69-76.

[19] Lindberg-Larsen, R., Møller, N., Schmitz, O., Nielsen, S., Andersen, M., Orskov, H. and Jørgensen, J.O. (2007) The Impact of Pegvisomant Treatment on Substrate Metabolism and Insulin Sensitivity in Patients with Acromegaly. The Journal of Clinical Endocrinology and Metabolism, 92, 1724-1728. http://dx.doi.org/10.1210/jc.2006-2276

[20] Campbell, P.J., Bolli, G.B. and Gerich, J.E. (1988) Prevention of the Dawn Phenomenon (Early Morning Hyperglycemia) in Insulin Dependent Diabetes Mellitus by Bedtime Intranasal Administration of Long Acting Somatostatin Analog. Metabolism, 37, 34-37. http://dx.doi.org/10.1016/0026-0495(88)90026-1

[21] Møller, N. and Jorgensen, J.O. (2009) Effects of Growth Hormone on Glucose, Lipid and Protein Metabolism in Human Subjects. Endocrine Reviews, 30, 152-177. http://dx.doi.org/10.1210/er.2008-0027

[22] Krusenstjerna-Hafstrøm, T., Clasen, B.F., Møller, N., Jessen, N., Pedersen, S.B., Christiansen, J.S. and Jørgensen, J.O. 
(2011) Growth Hormone (GH)-Induced Insulin Resistance is Rapidly Reversible: An Experimental Study in GH-Deficient Adults. The Journal of Clinical Endocrinology and Metabolism, 96, 2548-2557. http://dx.doi.org/10.1210/jc.2011-0273

[23] Perriello, G., De Feo, P., Torlone, E., Fanelli, C., Santeusanio, F., Brunetti, P. and Bolli, G.B. (1990) Nocturnal Spikes of Growth Hormone Secretion Cause the Dawn Phenomenon in Type 1 (Insulin Dependent) Diabetes Mellitus by Decreasing Hepatic (and Extrahepatic) Sensitivity to Insulin in the Absence of Insulin Waning. Diabetologia, 33, 52-59. http://dx.doi.org/10.1007/BF00586461

[24] Dux, S., White, N.H., Skor, D.A. and Santiago, J.V. (1985) Insulin Clearance Contributes to the Variability of Nocturnal Insulin Requirement in Insulin-Dependent Diabetes Mellitus. Diabetes, 34, 1260-1265. http://dx.doi.org/10.2337/diab.34.12.1260

[25] Jørgensen, J.O. (1991) Human Growth Hormone Replacement Therapy: Pharmacological and Clinical Aspects. Endocrine Reviews, 2, 189-207. http://dx.doi.org/10.1210/edrv-12-3-189

[26] Bright, G.M., Melton, T.W., Rogol, A.D. and Reaven, G.M. (1980) Failure of Cortisol Blockade to Inhibit Early Morning Increases in Basal Insulin Requirements in Fasting Insulin-Dependent Diabetics. Diabetes, 29, 662-664. http://dx.doi.org/10.2337/diab.29.8.662

[27] Skor, D.A., White, N.H., Thomas, L., Shan, S.D., Cryer, P.E. and Santiago, J.V. (1983) Examination of the Role of the Pituitary-Adrenocortical Axis, Counterregulatory Hormones, and Insulin Clearance in Variable Nocturnal Insulin Requirements in Insulin-Dependent Diabetes. Diabetes, 32, 403-407. http://dx.doi.org/10.2337/diab.32.5.403

[28] Xu, J. and Messina, J.K. (2009) Crosstalk between Growth Hormone and Insulin Signaling. Vitamins \& Hormones, 80, 125-153. http://dx.doi.org/10.1016/S0083-6729(08)00606-7

[29] Domenici, F.P., Argentino, D.P., Muñoz, M.C., Miquet, J.G., Sotelo, A.I. and Turyn, D. (2005) Influence of the Crosstalk between Growth Hormone and Insulin Signaling on the Modulation of Insulin Sensitivity. Growth Hormone \& IGF Research, 15, 324-336. http://dx.doi.org/10.1016/j.ghir.2005.07.001 